\title{
The comet 17P/Holmes 2007 outburst: the early motion of the outburst material ${ }^{\star}$
}

\author{
M. Montalto ${ }^{1,2}$, A. Riffeser ${ }^{2}$, U. Hopp ${ }^{1,2}$, S. Wilke ${ }^{2}$, and G. Carraro ${ }^{3}$ \\ 1 Max-Planck-Institute for Extraterrestrial Physics, Giessenbachstr., 85741 Garching b. Muenchen, Germany \\ e-mail: marco.montalto@unipd.it \\ ${ }^{2}$ Universitaets-Sternwarte der Ludwig-Maximilians-Universitaet, Scheinerstr.1, 81679 Muenchen, Germany \\ 3 ESO, Santiago
}

Received 22 November 2007 / Accepted 7 January 2008

\section{ABSTRACT}

\begin{abstract}
Context. On October 24, 2007 the periodic comet 17P/Holmes underwent an astonishing outburst that increased its apparent total brightness from magnitude $V \sim 17$ up to $V \sim 2.5$ in roughly two days. In this contribution we report on Wendelstein $0.8 \mathrm{~m}$ telescope (WST) photometric observations of the early evolution stages of the outburst.

Aims. We studied the evolution of the structure morphology and its kinematic and provide an estimate of the ejected dust mass. Methods. We analyzed 126 images of the comet in the BVRI photometric bands spread between October 26, 2007 and November 20, 2007. The bright comet core appeared to be separated from a quickly expanding dust cloud in all the data, and the bulk of the cloud was contained in the field of view of our instrument during the days soon after the outburst, allowing precise estimates both of the separation velocities of the two luminous baricenters and of the expansion velocity of the dust cloud. The ejected dust mass was derived on the basis of differential photometry on background stars occulted by the moving cloud.

Results. The two cores were moving apart from each other at a relative, projected constant velocity of $(9.87 \pm 0.07)$ arcsec/day $\left(0.135 \pm 0.001 \mathrm{~km} \mathrm{~s}^{-1}\right)$. In the inner regions of the dust cloud we observed a linear increase in size at a mean constant velocity of $(14.6 \pm 0.3) \mathrm{arcsec} /$ day $\left(0.200 \pm 0.004 \mathrm{~km} \mathrm{~s}^{-1}\right)$. Evidence of a radial velocity gradient in the expanding cloud was also found. Our estimate for the expanding coma's mass was approximately $10^{-2}-1$ comet's mass, implying a significant disintegration event. Conclusions. We interpret our observations in the context of an explosive scenario that was more probably triggered by some internal instability processes rather than by an impact with an asteroidal body. Due to the peculiar characteristics of this event, further observations and investigations are necessary to bring the nature of the physical processes that determined it to light.
\end{abstract}

Key words. comets: individual: 17P/Holmes - solar system: general

\section{Introduction}

In this Letter, we concentrate on analyzing the outburst of comet 17P/Holmes which occurred on October 24, 2007 (Santana 2007). We provide an overview analysis of the early evolution phases of the surrounding cometary environment in the period between October 26 and November 20, 2007 considering the global structure of the expanding material and its kinematic properties and inferring the ejected dust mass. Comet brightenings are well-documented in the literature, and in general they may be associated with evident cometary nuclear fragmentation (see e.g. Sekanina et al. 2002, and references therein). Despite their recurrence, the magnitude and the characteristics of the outbursts on comet $17 \mathrm{P} /$ Holmes largely justify its longstanding reputation in the annals of astronomy (Barnard 1896).

The periodic comet $17 \mathrm{P} /$ Holmes was discovered on November 6, 1892 by E. Holmes in London, during an outstanding brightness increase, followed by another similar event on January 16, 1893. When discovered by Mr. Holmes, the comet was around 5 months past perihelion ( $T=$ June 13). On October 24, 2007, around 6 months after the last perihelion passage ( $T=$ May 4,2007$)$, the comet underwent a phenomenon similar to those observed more than one hundred years ago. The heliocentric distance of the object at the time of the two major

^ Based on observations taken at the Wendelstein $0.8 \mathrm{~m}$ telescope. events was around $\Delta_{\odot}=2.39 \mathrm{AU}$ in 1892 and $\Delta_{\odot}=2.44 \mathrm{AU}$ in 2007 , and the orbital inclination remained substantially unaltered during this period $\left(i \sim 20^{\circ}\right)$. This Letter is structured in the following way. In Sect. 2, we present the observations and the data reduction method. In Sect. 3, we discuss the morphological evolution of the expanding structure. In Sect. 4, we calculate the separation velocities between the comet and the center of the dust cloud, as well as the expansion velocity of the cloud. In Sect. 5, we estimate the ejected dust mass. Finally in Sect. 6, we sum up our results.

\section{Observations and reduction of the data}

The observations were acquired on October 26/28/29/31, 2007 and November 2/5/20, 2007. A total number of 126 images were analyzed in the $B V R I$ filters, and an overview of the observations is shown in Table 1. The images were acquired using the MONICA CCD camera, at the Cassegrain focus of the $0.8 \mathrm{~m}$ Wendelstein telescope, with a scale of $0.5 \mathrm{arcsec} /$ pix and a field of view of around $8 \times 8$ arcmin. The pre-reduction was done using standard reduction software (MUPIPE) specifically developed at the Munich Observatory for the MONICA CCD camera (Gössl \& Riffeser 2002). The weather conditions were in general clear for all our observing nights with the exception of night 26 , which was cloudy. Otherwise we were able to acquire 1 image 
Table 1. Number of images of 17P/Holmes taken during the different observing nights in the $B V R I$ filters.

\begin{tabular}{ccccc}
\hline \hline Date & $B$ & $V$ & $R$ & $I$ \\
\hline $26 / 10 / 2007$ & - & - & 1 & - \\
$28 / 10 / 2007$ & 6 & 6 & 9 & 11 \\
$29 / 10 / 2007$ & 10 & 5 & 10 & 10 \\
$31 / 10 / 2007$ & 10 & - & 12 & 13 \\
$02 / 11 / 2007$ & 4 & 3 & 5 & - \\
$05 / 11 / 2007$ & - & - & 5 & - \\
$20 / 11 / 2007$ & - & - & 6 & - \\
\hline
\end{tabular}

in the $R$ band that night and to use it for analyzing the kinematic properties of the cloud described in Sect. 4. The exposure times varied between 40 and $200 \mathrm{~s}$ for the whole dataset.

During night 28, the photometric conditions were both good and stable over the whole night, and we thus acquired some images of the Landolt standard field PG0918 in all our filters. We obtained 2 images in the $B, V, R$ bands and 4 images in the $I$ band. These data were reduced in exactly the same manner as the scientific observations (see also Sect. 5). The magnitudes of the standard stars were reported to 1 airmass and 1 arcsec and finally calibrated to the Landolt photometric system, thanks to the Stetson Photometric Standard Fields ${ }^{1}$ data of PG0918. We found a total of 26 common stars between our catalog and the one provided by Stetson, and obtained the following calibration equation for the $R$ band against the $V-R$ color:

$R-r=-0.07( \pm 0.02)(V-R)+23.18( \pm 0.01)$.

The rms residual of the fit with respect to the best-fitting leastsquare linear model was $\sim 0.02 \mathrm{mag}$. This result gives an idea of our photometric precision during that night, and it will be useful in Sect. 5 in the discussion of the ejected mass estimate.

\section{The evolution of the morphological structure}

To show the evolution of the morphological structure of the object, we selected one representative image in the $R$ band for each night in our dataset. These images were scaled to the same exposure time, airmass, and intensity range, in order to properly sample the surface brightness of the coma in the whole dataset. The lowest intensity level was chosen close to the lowest counts we got on the last image of our dataset, while the highest was chosen close to the coma's peak intensity of the image taken on October 28, 2007. We did not process the image acquired on October 26, 2007 in this way, because it was too noisy. The images were then normalized to the lowest intensity level. Finally, we displayed the images codified on a logarithmic color scale, as shown in Fig. 1. We overplotted the isophote intensities levels to each panel to highlight the structure of the inner part of the cloud.

The most interesting feature in Fig. 1 is the presence of two bright cores (the comet core being towards the upper left side). These two cores were increasingly separating during the observing period and appeared elongated towards each other, indicating an exchange of mass. Moreover, the expansion of the global structure is evident from the sequence of images. We also displayed the intensity value of the closest isophote to the cloud's center and of the adjacent one in the outwards direction. The

\footnotetext{
1 http://www3. cadc-ccda.hia-iha.nrc-cnrc.gc.ca/ community/STETSON/standards/
}
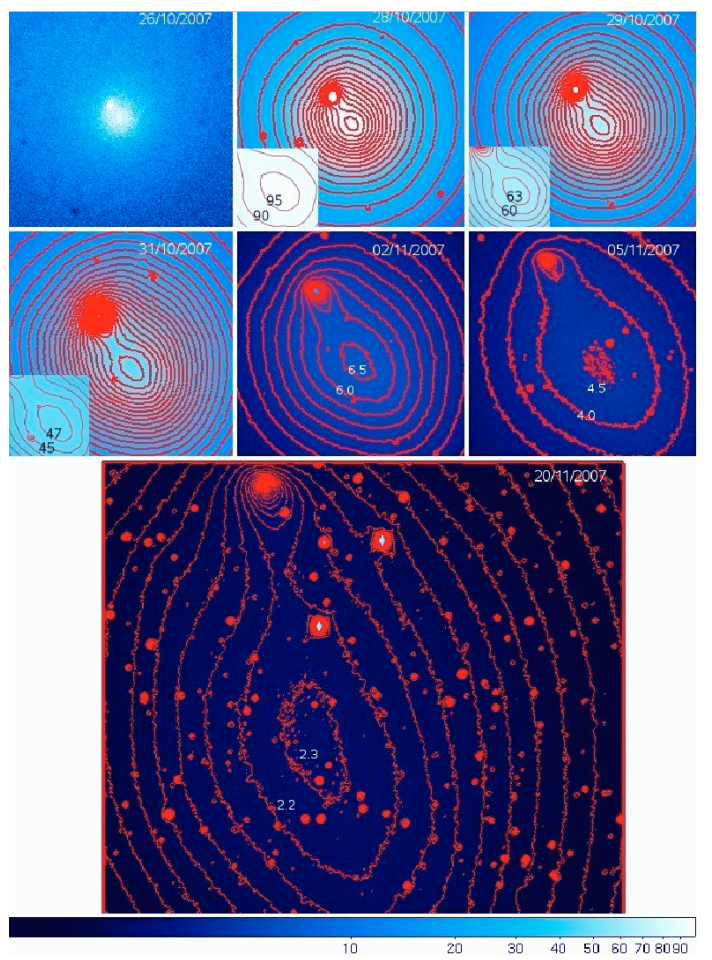

Fig. 1. Snapshot of $R$ band images acquired during each night of our observing run, showing the evolution of comet 17P/Holmes' morphological structure. Each image has been scaled to the same exposure time, airmass, and intensity range and displayed on a logarithmic color scale, codified by the bottom color bar. The top 6 images are zoomed inside the inner regions of the object and have a field of view of $2.2 \times 2.2 \mathrm{arcmin}$, whereas the last one $8 \times 8 \mathrm{arcmin}$. Two cores are visible in all the images, the comet nucleus (on the upper left side) and the core of the coma, rapidly separating during the observing period. The values reported for the coma's innermost isophotes of each panel (or in the close-up views of 28/29/31 nights) show the surface brightness decrement of the coma due to its rapid expansion.

exact values given by the isophotes depend on the adopted number of isophotes in each panel, which also determines the intensity step between them. We set the number of isophotes to approximately provide the same sampling of the inner regions of the cloud. Thus, the quoted numbers must be considered as indicative of the relative surface brightnesses of the cloud on the different nights. The intensity of the innermost isophote of night 2007 Oct. 28 was around 2 orders of magnitude larger with respect to the correpondent isophote's intensity of night 2007 Nov. 20, and the intensity step between the isophotes was also 1 order of magnitude larger in the first case with respect to the second. We emphasize that there is no way to significantly change these conclusions by adopting different isophotal mapping criteria, as we accurately verified. Actually, both these results are the consequence of the rapid expansion of the dust cloud, which determines a steady decrease in its surface brightness, and a more homogeneous light distribution in the more evolved and less concentrated phases.

\section{Kinematic of the expanding materials}

In this section we derive the separation velocity of the two bright baricenters and the dust cloud's expansion velocity. The comet's center appeared in all our images as a point-like source, so the peak's position was derived with a Gaussian fitting algorithm, as for stellar objects. The light distribution of the dust cloud was 

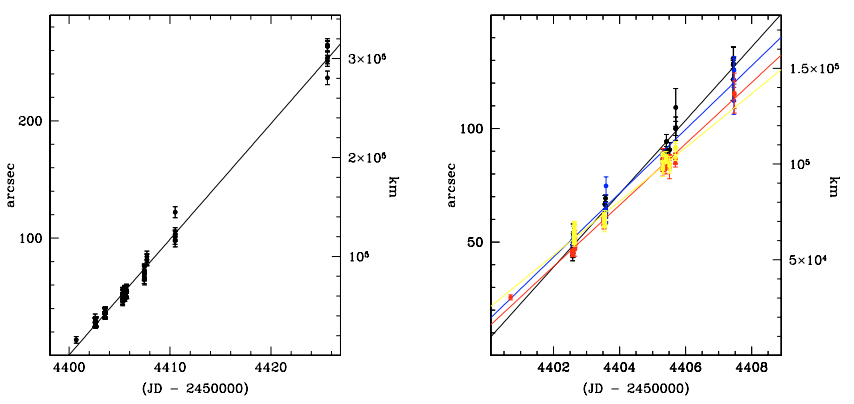

Fig. 2. Left: angular projected distance between the centroid of the two bright baricenters, for all the images in our dataset, against the JD of the observations. The continuous line denotes the best fit linear model. On the left hand $y$-axis values are in arcsec, whereas on the right hand $y$-axis values are in $\mathrm{km}$. Right: $\sigma$ of the expanding cloud Gaussian core against JD. Different colors indicate measurements obtained in different bands: $B$ in black, $V$ in blue, $R$ in red and $I$ in yellow. The best fit linear models in each band are represented by the continuous lines.

extended over a much larger area and was not Gaussian overall, but we found that the inner part could be always represented by a Gaussian. We thus fit a bidimensional Gaussian using the light distributions along the $x$ and $y$ axis. An initial guess for the centroid was provided with a maximum finding algorithm run in a small region around the peak $(\sim 30 \mathrm{arcsec})$. Then a least-square fit provided the refined position for the centroid and the $\sigma_{x}$ and $\sigma_{y}$ of the fitting Gaussians. In Fig. 2 (left panel) we plotted the two bright cores' projected distances against the Julian date (JD) of the observations. The correction for the Earth's variable distance has been taken into account, although it is negligible. We obtained a uniform increasing separation with a mean velocity of $(9.87 \pm 0.07)$ arcsec/day $\left(0.135 \pm 0.001 \mathrm{~km} \mathrm{~s}^{-1}\right)$. The uncertainties are dominated by the errors in the diffuse cloud centroids. In the right panel of Fig. 2, we report the $\sigma$ of the diffuse clouds (actually the mean of the $\sigma_{x}$ and $\sigma_{y}$ ) as a function of the JD. We did not include the last two nights in this figure, because the cloud was too expanded. Two images acquired on night 2007 Nov. 2 in the $R$ band were also excluded because of the large offset of the cloud's centroid with respect to the image's center. The errorbars in this figure were calculated as the difference of the $\sigma$ along the $x$ and $y$ axes. The different colors in the figure codify observations obtained in the different photometric bands. Also in this case we observed a linear increase in size with a mean velocity of $(14.6 \pm 0.3)$ arcsec/day $\left(0.200 \pm 0.004 \mathrm{~km} \mathrm{~s}^{-1}\right)$. Repeating the calculation separately for the different bands gave the results in Table 2. These mean values and their errors were obtained using a weighted least-square algorithm. The different bands allow exploration of the different layers of the expanding cloud, more external in the $B$ and deeper in the redder bands. Thus, this result can be interpreted as evidence of a radial velocity gradient in the expanding cloud. For each couple of adiacent bands, we considered the ratio between the difference of the expansion velocities reported in Table 2 and the difference in the correspondent $\sigma$ values given by the leastsquare fitting models for the night 2007 Nov. 2, for which we obtained the largest separation among the expanding shells. Taking the mean of these values, we obtained an estimate of the radial velocity gradient during that night equal to $(0.3 \pm 0.2) 10^{-5} \mathrm{~s}^{-1}$, which means an increase of around $0.3 \mathrm{~cm} / \mathrm{s}$ every $\mathrm{km}$ going from the center to the surface of the coma.

All these kinematic observations could be explained in the context of an explosive scenario. While, as a consequence of the explosion, a part of the cometary nucleus was disintegrated
Table 2. The derived expansion velocities in arcsec/day along with their uncertainties for the expanding dust cloud in the different bands. In parenthesis we report the corresponding values in $\mathrm{km} \mathrm{s}^{-1}$.

\begin{tabular}{cccc}
\hline \hline Filter & Expansion vel. & \pm & err \\
\hline$B$ & $16.2(0.222)$ & & $0.5(0.007)$ \\
$V$ & $14.0(0.193)$ & & $0.6(0.009)$ \\
$R$ & $13.5(0.185)$ & & $0.2(0.003)$ \\
$I$ & $11.9(0.163)$ & $0.4(0.005)$ \\
\hline
\end{tabular}

and the material was outflowed in all directions resulting in the sperically symmetric expanding coma, and the survived nucleus received a kick separating from the coma's center with the observed projected velocity. The expansion velocity of a particle of radius $a$ during an explosive event is proportional to $a^{-1}$ (see e.g. the discussion in Tozzi et al. 2007) implying a higher expansion velocity for the smaller, less massive particles than for the larger and more massive ones, which resulted in the observed velocity gradient in the expanding cloud.

The formation of spherical expanding envelopes around the cometary nucleus of $17 \mathrm{P} /$ Holmes was observed for both the events in November 1892 and January 1893. Bobrovnikoff (1943) compared the observations by different authors between 16 and 22 January 1893, deriving a uniform expansion velocity of $(0.54 \pm 0.03) \mathrm{km} \mathrm{s}^{-1}$, similar to what we found here. The sudden brightness variations, the formation of spherical dust envelopes with similar kinematic properties, the correspondence in the orbital phase at the instant of the major outbursts noted in Sect. 1, point towards a common mechanism at the base of the observed phenomena. Moreover, the distance from the ecliptic plane was around $0.8 \mathrm{AU}$ in both cases. Given that, although it cannot be completely excluded, it seems unlikely that the abovementioned explosive mechanism was triggered by an asteroidal impact, making it more probably an internal instability process.

\section{The ejected mass estimate}

In the following we provide an estimate of the ejected dust mass during the outburst, through the extinction produced by the dust cloud on the surrounding background stars. We selected two well-exposed, seeing images taken on October 28, 2007 in the $R$ filter. These images had the largest time separation $(2 \mathrm{~h})$ in the whole dataset, among equal filter images acquired during the same observing night. Thus, they provided the largest apparent motion of the cloud on the sky plane. At the same time, this temporal difference is small enough to avoid the expansion of the cloud changing the extinction map significantly, thereby allowing a homogeneous comparison of the two images. Finally, day 28 was close enough to the outburst to allow the dust cloud to fit well inside our field of view. We performed PSF fitting photometry with DAOPHOT/ALLSTAR (Stetson 1987) and rejected all the stars with $\chi>1.5$ and any absolute sharp values bigger than 1 . These parameters and selection criteria allowed those objects to be excluded for which a reliable fit of the stellar model could not be performed, because close to saturation, or close to bad pixels, and to reject non-stellar sources such as cosmic rays, background galaxies, or spikes of saturated stars. The sky background in these images was estimated locally around each star in an annular region comprised 1.5 and 3.5 arcsec from the stars' centroids. This region was selected after performing different tests looking for the best subtraction of the analyzed stars. The modal value of the pixel counts inside the selected annular region was considered as the estimate of the sky background. The 

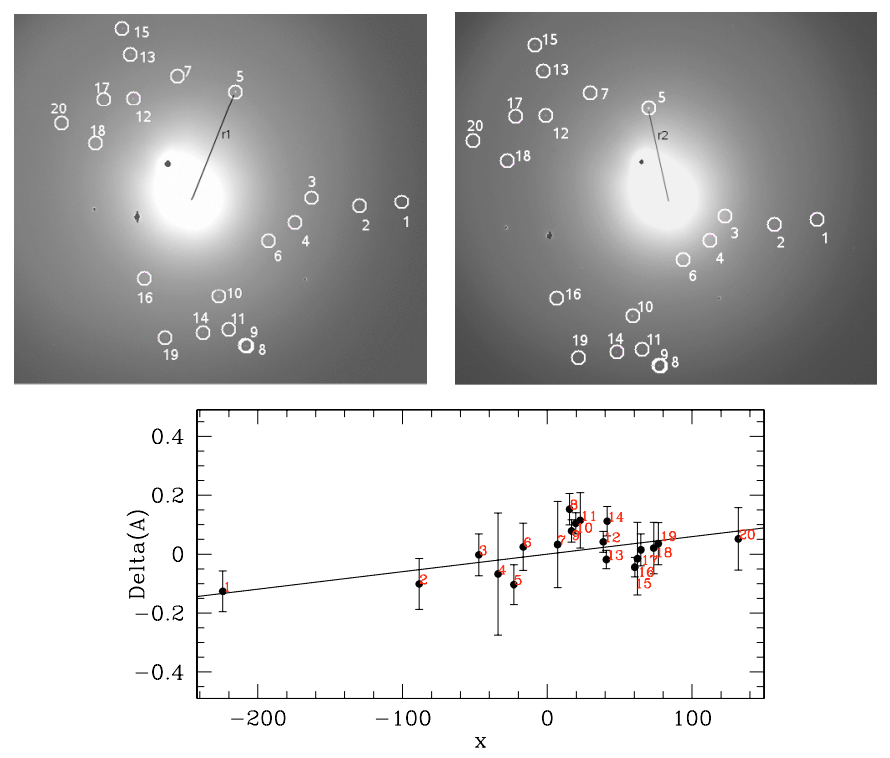

Fig. 3. Top: spatial distribution of the 20 background stars used in Sect. 5 to probe the differential extinction produced by the coma, for the two images acquired on October 28, 2007. The image on the left was taken $2 \mathrm{~h}$ before the right one. The selected stars are indicated by white circles. The associated numeration corresponds to the one in the lower panel, allowing each star to be related to the correspondent measured differential extinction. Bright sources were saturated (black regions), as well as was the center of the cometary nucleus. The black lines indicate the distances, referred to in Sect. 5 as $r_{1}$ and $r_{2}$, of one star with respect to the estimated coma's centers. Bottom: the correlation between the differential extinctions in the $R$ band $(\Delta A)$ and the $x$ parameter (see text for details).

photometry was extracted in a circular region centered on the stars' centroids with radius equal to $1.3 \mathrm{arcsec}$, which corresponded to the seeing value in both images. The derived magnitudes were then reported to 1 airmass and $1 \mathrm{~s}$ of exposure time for both the images. We then considered all the common objects with a radial distance from the center of the comet $>25$ arcsec and with radial distance from the center of the cloud $<3$ arcmin in both the images. The inner limit was needed to avoid the photometry of the stars being biased by the luminous core of the comet. The outer limit was chosen to avoid detector border effects, accounting for the position of the center of the cloud in both the images. At the end, we obtained a catalog of 20 stars spread inside the analyzed region of the cloud; see Fig. 3.

To demonstrate the radial dependence of the extinction from the center of the cloud, we assumed a uniform and homogeneous, spherically symmetric mass distribution. Thus, because $r$ is the position of one star with respect to the cloud's center, we can express the optical depth of the cloud at the distance $r$ as

$\tau(r)=\sigma_{\mathrm{d}} n 2 \sqrt{R^{2}-r^{2}}$

where $2 \sqrt{R^{2}-r^{2}}$ is the length of the segment of sphere with projected distance $r$ with respect to the cloud's center, $R$ is the radius of the cloud, $\sigma_{\mathrm{d}}$ the cross section of the dust grains in a given observing band, and $n$ the number density of the dust particles. Considering two different positions $r_{1}$ and $r_{2}$ of a generic star in the first and second images with respect the cloud's center, we can express the differential extinction $\Delta \mathrm{A}$ produced by the cloud in the two positions as

$\Delta A=m_{1}-m_{2}=-2.5 \log \left(\frac{I_{1}}{I_{2}}\right)=-2.5 \log \left[\frac{\mathrm{e}^{-\sigma_{\mathrm{d}} n 2 \sqrt{R^{2}-r_{1}^{2}}}}{\mathrm{e}^{-\sigma_{\mathrm{d}} n 2 \sqrt{R^{2}-r_{2}^{2}}}}\right]$, which implies

$$
\begin{aligned}
\Delta A & =\left(2.5 \sigma_{\mathrm{d}} n 2 \log e\right)\left(\sqrt{R^{2}-r_{1}^{2}}-\sqrt{R^{2}-r_{2}^{2}}\right) \\
& =\alpha\left(\sqrt{R^{2}-r_{1}^{2}}-\sqrt{R^{2}-r_{2}^{2}}\right)
\end{aligned}
$$

where $m_{1}, I_{1}$ and $m_{2}, I_{2}$ are the observed magnitudes (intensities) of the star at positions $r_{1}$ and $r_{2}$, respectively. As easily recognized, this model satisfies the required symmetry conditions with respect to $r_{1}$ and $r_{2}$. Actually, if $r_{1}$ is equal to $r_{2}$, the differential extinction should be zero. Morever, if we consider two stars with initial $r_{1}, r^{\prime}{ }_{1}$ and final $r_{2}, r_{2}^{\prime}$ positions, which satisfy the condition $r_{1}=r^{\prime}{ }_{2}$ and $r_{2}=r^{\prime}{ }_{1}$, the resulting differential extinctions for the two stars should be equal and opposite in sign. In particular, $\Delta A$ should be positive when $r_{1}<r_{2}$ (the star is moving away from the cloud center, and thus is less extincted in $r_{2}$ with respect to $r_{1}$ ), and negative when $r_{1}>r_{2}$ (the star is going towards the cloud center). This implies that the angular coefficient $\alpha$ in Eq. (3) should be positive. The value of $\alpha$ gives the extinction for unitary length in the given observing band. Therefore, we fit to the observed differential extinctions the model

$\Delta A=\alpha x+\beta$

where $x=\sqrt{R^{2}-r_{1}^{2}}-\sqrt{R^{2}-r_{2}^{2}}$, and the $\beta$ coefficient was included to account for residual constant zero points between the two images. The result, after subtracting the constant zero point, is shown in the bottom panel of Fig. 3. We found a positive correlation between the differential extinction $\Delta A$, and the $x$ parameter, as expected. The derived value of the $\alpha$ coefficient was $(0.0006 \pm 0.0002) \mathrm{mag} / \mathrm{pix}$, where a pixel corresponds to around $592 \mathrm{~km}$ at the distance of the comet. This value has been obtained assuming a radius $R$ for the cloud of 3 arcmin $(\sim 3.5 \sigma$ for that night for the luminous distribution analyzed in Sect. 4). Increasing the radius to $4 \operatorname{arcmin}(\sim 5 \sigma)$ implies a value of $\alpha=(0.0012 \pm 0.0004) \mathrm{mag} / \mathrm{pix}$.

The most important assumptions of the model are the dust cloud's spherical geometry and the homogeneous and uniform mass distribution. The geometry of the cloud was wellconstrained by the observations and the analysis presented in the previous sections. As for the second hypothesis, it allowed expression of the optical depth in a straightforward and convenient way, as shown in Eq. (1), and ultimately implies the linear dependence of the observed differential extinctions on the geometrical factor $x$. A strong deviation from that assumption would imply a strong deviation from the linear model prediction, which is not observed (Fig. 3). The scatter in that relation ( 0.06 mag) is greater than the scatter derived from the calibration of the Landolt standard field discussed in Sect. 2 ( $\sim 0.02 \mathrm{mag})$. Otherwise, in the science images, the surface brightness of the coma determined a background around 10 times larger than in the Landolt images, which explains the factor of 3 increase in the scatter. The radial velocity gradient of the expanding material discussed in Sect. 4 points away from the uniform mass distribution hypothesis. Otherwise, as shown in the right panel of Fig. 2, during night 28 the expanding shells were certainly more concentrated than in the later evolved phases since closer that night to the outburst. In conclusion, it is reasonable to believe that, on the chosen night, the material was not far from being uniformly distributed and homogeneously mixed, and we considered this hypothesis as a good approximation of the structural properties of the observed coma. Therefore, the dominant factor that explains the observations is related to the cloud's geometrical structure, in the sense that the observed differential 
extinctions were determined by the variable quantity of mass over the cloud's different line of sights probed by the background stars (directly implied by its spherical structure). This is demonstrated overall by the observed linear dependence of the differential extinction on the geometrical factor $\mathrm{x}$ and by the positive value of the $\alpha$ coefficient, also predicted by the model.

To derive the coma's mass, we considered spherical dust grains with a mean density $\rho_{\mathrm{d}}\left(=2.5 \mathrm{~g} / \mathrm{cm}^{3}\right)$ and a "typical" dimension $r_{\mathrm{d}}$ (and thus mass $m_{\mathrm{d}}=4 / 3 \pi r_{\mathrm{d}}^{3} \rho_{\mathrm{d}}$ ). Thanks to the definition of the $\alpha$ parameter in Eq. (3), the mass of the cloud $M$ can be expressed through the formula

$$
\begin{aligned}
M & =\frac{4}{3} \pi R^{3} m_{\mathrm{d}} n=\frac{4}{3} \pi R^{3}\left(\frac{4}{3} \pi r_{\mathrm{d}}^{3} \rho_{\mathrm{d}}\right)\left(\frac{\alpha}{5 \sigma_{\mathrm{d}} \log e}\right) \\
& =\frac{16 \pi}{45 \log e} R^{3} r_{\mathrm{d}} \rho_{\mathrm{d}} \alpha \simeq R^{3} r_{\mathrm{d}} \rho_{\mathrm{d}} \alpha
\end{aligned}
$$

where the grain's cross-section was taken equal to the geometrical cross-section $\sigma_{\mathrm{d}}=\pi r_{\mathrm{d}}^{2}$ and the numerical factor is $\sim 2.6$. We varied the value of $r_{\mathrm{d}}$ inside a range of characteristic grain dimensions, 0.005-1 $\mu \mathrm{m}$ (Mathis et al. 1977), and the dimension of the cloud $R$ between 3-4 arcmin. The derived estimate for the coma's mass was between $10^{12}-10^{14} \mathrm{~kg}$. Snodgrass et al. (2006) provides the most recent values for this comet's dimension and density. In particular, with their time-series photometry they obtained a value for the effective radius of the nucleus (Russel $1916)$ of $(1.62 \pm 0.01) \mathrm{km}$. Even if accurate, this estimate is based on the assumption that the geometrical albedo was equal to $A_{R}=0.04$. Furthermore, they derived a comet's minimum density equal to $\rho=0.09 \mathrm{~g} / \mathrm{cm}^{3}$. Assuming thus a range of possible densities $\left(\rho=0.1-1 \mathrm{~g} / \mathrm{cm}^{3}\right)$ and albedos $\left(A_{R}=0.01-0.1\right)$ and using Eq. (1) in Snodgrass et al. (2006) to derive the corresponding effective radii, we obtained a total nuclear mass between $10^{12}$ and $10^{14} \mathrm{~kg}$. Given the uncertainty range in our mass estimate, we concluded that the probable value of the expanding coma's mass resulting from the outburst event ranged between $10^{-2}$ and 1 comet's mass.

There are different factors that could affect our results. Dust grains in cometary ejecta typically span a range of different dimensions and optical properties (see e.g. Lisse et al. 2007; Tozzi et al. 2007). Both the assumption on the uniform and homogeneous mass distribution and the lack of specific observations able to constrain the dust population characteristics for this particular object together justify our simplified approach to summarizing the cloud's grain content, assuming a "typical" grain dimension and the corresponding geometrical cross-section. Another possible drawback in our coma's mass estimate regards the preoutburst activity of the comet. During the most recent observations of the comet obtained before the outburst (Snodgrass et al. 2006), the object appeared to be inactive, although the heliocentric distance at that time was $\sim 4.66 \mathrm{AU}$, whereas at the outburst it was $\sim 2.44$ AU. Moreover, it seems probable that the explosive event described in this work largely overcame the common activity of the comet. Finally, the stars used to probe the differential extinction produced by the coma (Fig. 3), were well-distributed inside the coma, avoiding the region close to the comet's nucleus, and thus more closely reflecting the contribution to the differential extinction of the material coming directly from the explosion.
Despite these approximations, the result presented in Eq. (5), has the advantage to enlight the dependence among the total mass of the cloud, the cloud's geometrical, composition properties and the observed differential extinctions, and of providing an estimate for the expanding coma's mass that points towards a consistent disintegration phenomenon, as suggested by the large outburst event.

\section{Conclusions}

In this Letter we analyzed the early phases of the 2007 October 24 outburst comet 17P/Holmes. We acquired $B V R I$ photometric images at the Wendelstein $0.8 \mathrm{~m}$ telescope between October 26, 2007 and November 20, 2007. We observed a spherically symmetric dust cloud moving away from the comet nucleus with a mean projected constant velocity of $(9.87 \pm 0.07)$ arcsec/day $\left(0.135 \pm 0.001 \mathrm{~km} \mathrm{~s}^{-1}\right)$, while the dust cloud was expanding with a mean constant velocity of $(14.6 \pm 0.3)$ arcsec/day $\left(0.200 \pm 0.004 \mathrm{~km} \mathrm{~s}^{-1}\right)$. These results agree with those obtained during past outbursts of this comet. Evidence of a gradient in the expansion velocity of the dust cloud was also found with the velocity increasing towards the external regions. Finally, performing differential photometry on background stars occulted by the moving cloud, and assuming a uniform and homogeneous spherical mass distribution, we derived a value of $10^{12}-10^{14} \mathrm{~kg}$ for the coma's mass, around $10^{-2}-1$ of the comet's mass. We interpreted our observations in the context of an explosive event, probably caused by some internal instability processes rather than by an asteroidal body's impact. Various mechanisms have been proposed in the past to explain the comets' splitting, involving tidal, thermal, and rotational forces (Sekanina 1997). These processes could have played an important role in the event discussed here, although some specific characteristics allow us to consider it as more peculiar. For example, the separation velocities of the splitting components are generally a few $\mathrm{m} / \mathrm{s}$, while in this case we found a projected relative velocity around 2 orders of magnitude greater. The outburst itself represents the largest apparent brightness increase ever observed for a comet. In conclusion, we underline the importance of considering other observational results in order to accurately characterize this event and to provide a more insightful view of its enigmatic and still not well-understood nature.

Acknowledgements. We warmly thank the anonymous Referee for the helpful comments and suggestions allowing significant improvement to this Letter.

\section{References}

Barnard, E. E. 1896, ApJ, 3, 41

Bobrovnikoff, N. T. 1943, PA, 51, 542

Gössl, C. A., \& Riffeser, A. 2002, A\&A, 381, 1095

Lisse, C. M., Kraemer, K. E., Nuth, J. A., Li, A., \& Joswiak, D. 2007, Icarus, 191,223

Mathis, J. S., Rumpl, W., Nordsieck, K. H. 1977, ApJ, 217, 425

Russell, H. N. 1916, ApJ, 43, 173

Santana, A. H. 2007, IAU Circ., 8886

Sekanina, Z. 1997, A\&A, 318, 5

Sekanina, Z., Jehin, E., Boehnhardt, H., et al 2002, ApJ, 572, 679

Snodgrass, C., Lowry, S. C., \& Fitzsimmons, A. 2006, MNRAS, 373, 1590

Stetson, P. B. 1987, PASP, 99, 191

Tozzi, G. P., Boehnhardt, H., Kolokolova, L., et al. 2007, A\&A, 476, 979 